\title{
Effects of Enhanced External Counter-Pulsation therapy on QT Dispersion in Patients with Heart Failure
}

\author{
Vahid Ghasem Amooeian ${ }^{1}$, Kaveh Hosseini ${ }^{2}$, Ali Bozorgi ${ }^{3}$, Ehsan Rashidi ${ }^{4}$, Maryam Shahali Ramshe ${ }^{5}$, \\ Farzad Masoudkabir $^{6}$, Mehdi Bayati ${ }^{7}$, Ali Vasheghani-Farahani ${ }^{* *}$
}

\begin{abstract}
Background: Enhanced External Counter Pulsation (EECP) is a FDA approved noninvasive technique that reduces symptoms of certain heart related diseases in patients. It simulates exercise training condition for patients unable to intercept an actual training session. It also hypothetically affects ECG markers such as QT dispersion in the same way that exercise training does.

Present study is mainly focused on evaluating several ECG parameters including PR, QRS and QT interval before and after using EECP in CHF patients in order to ultimately investigate the impression of EECP on dropping the risk of developing arrhythmias.

Method: Participants of this cross-sectional study was consist of 26 patients ( 21 men vs. 5 women) who had indication for EECP, fulfilled the entrance criteria, and were suffering from functional class II or III in NYHA classification and left ventricular Ejection Fraction $(\mathrm{EF}) \leq 35 \%$. Mean age of subjects was $56 \pm 2.2$ years.

All patients underwent at least 4 ECGs (weekly) and 4 weeks of EECP program. Changes in certain ECG parameters including QRS duration as well as PR and QT intervals were assessed during 4 weeks of using EECP technique for each individual. Baseline ECG was compared with 4th week ECG.

Result: No statistically differences were found between mean QRS duration $(\mathrm{P}=$ 0.292), mean PR interval $(\mathrm{P}=0.82)$, and mean $\mathrm{QT}$ dispersion $(\mathrm{P}=0.89)$ before and after 4 weeks of EECP therapy.

Conclusion: Based on this cross sectional study, 4 weeks of EECP did not have any significantly impact on ECG parameters. However, EECP might improve patient's functional capacity.
\end{abstract}

Keywords: Enhanced External Counter-Pulsation (EECP); QT dispersion; Heart failure; PR interval

\section{Introduction}

The growing number of patients with symptomatic heart failure, motivates physicians and researchers to expand new therapeutic methods in order to enhance cardiac contractility of the patients and lower their peripheral vascular resistance which results in better LV function ${ }^{[1]}$. Enhanced External Counter Pulsation (EECP) is a FDA approved noninvasive technique that reduces heart failure and angina pectoris symptoms in patients ${ }^{[2,3]}$. Reducing myocardial oxygen demand, increasing venous return as well as cardiac output, and improving the endothelial function are considered as different mechanisms in which EECP can enhance the heart efficacy ${ }^{[4,5]}$. Previous literature have demonstrated that patient's functional class based on NYHA classification significantly improves by using EECP technique ${ }^{[6]}$. QT dispersion is a measure of inter-lead QT interval variability in the surface ECG, described by Campbell et $\mathrm{al}^{[7]}$. The QT dispersion which is defined as the difference of maximal and minimal QT interval duration, reflects the regional differences in ventricular recovery time $^{[8,9]}$. Recent studies have shown that patients with increased QT dispersion are at greater risk for developing ventricular arrhythmias and sudden cardiac death in heart failure and coronary artery disease ${ }^{[10,11]}$. The study in Nara medical university have

\section{Open Access}

Research Article

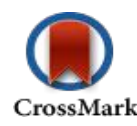

${ }^{1}$ Student of Medicine, Students' Scientific Research Center (SSRC), Cardiac Primary Prevention Research Center (CPPRC), Tehran University of Medical Sciences, Tehran, Iran

${ }^{2}$ Cardiology resident, Master of public health, Tehran heart center, Tehran University of medical sciences, Cardiac Primary Prevention Research Center (CPPRC), Department of cardiology, Tehran Heart Center, Tehran, Iran ${ }^{3}$ Assistant professor of cardiology, Department of cardiology, Tehran Heart Center, Karegar Shomali St., Jalal al-Ahmad Cross, Tehran, Iran

${ }^{4}$ Student of Medicine, Students' Scientific Research Center (SSRC), Tehran University of Medical Sciences, Tehran, Iran

${ }^{5}$ Scientific Research Center (SSRC), Cardiac Primary Prevention Research Center (CPPRC), Tehran University of Medical Sciences, Tehran, Iran

${ }^{6}$ Assistant Professor of Cardiology, Cardiac Primary Prevention Research Center (CPPRC), Tehran Heart Center, Tehran University of Medical Sciences, Tehran, Iran ${ }^{7}$ Student of Medicine, Students' Scientific Research Center (SSRC), Cardiac Primary Prevention Research Center (CPPRC), Tehran University of Medical Sciences, Tehran, Iran

${ }^{8}$ Associated professor of cardiology, Cardiac Primary Prevention Research Center (CPPRC), Department of cardiology, Tehran Heart Center, Karegar Shomali St., Jalal al-Ahmad Cross, Tehran, Iran

Received date: June 19, 2017

Accepted date: July 28, 2017

Published date: August 04, 2017

\section{"Corresponding author:}

Ali Vasheghani-Farahani, Assistant professor of cardiology, Cardiac Primary Prevention Research Center (CPPRC), Department of cardiology, Tehran Heart Center, Karegar Shomali St., Jalal al-Ahmad Cross, Tehran, Iran, Tel: +9821-88029257; Fax: +98-21-88029256;

E-mail: avasheghani@sina.tums.ac.ir

Citation: Ali Vasheghani-Farahani, et al. Effects of Enhanced External Counter-Pulsation therapy on QT Dispersion in Patients with Heart Failure. (2017) J Heart Cardiol 3(2): 1- 4.

DOI: 10.15436/2378-6914.17.1577 
demonstrated the benefits of exercise training on sympathovagal balance which leads to reduced QTcd ${ }^{[12]}$. EECP technique simulates exercise training condition for patients unable to intercept an actual training session and hypothetically affects ECG markers in the same way that exercise training does. Assessing the QT dispersion might be useful in arrhythmia risk assessment and evaluating the effectiveness of antiarrhythmic drug therapy ${ }^{[8]}$. As a matter of fact, clinical interventions in order to reduce QT dispersion might have a favorable impact on clinical outcomes. The aim of present study is to evaluate several ECG parameters including PR interval, QRS and QT interval, in heart failure group before and after using EECP. Indeed, we aim to investigate the impression of EECP on dropping the risk of developing arrhythmias in CHF patients.

\section{Methods and Material}

This cross sectional study was conducted in Tehran Heart Center from May 2015 to August 2016. All participants had symptomatic heart failure despite maximum medical (pharmacological) treatments. Selection method has been depicted in Figure 1.

Mean QRS duration was measured 80.76 milliseconds (ms) before EECP and $82.3 \mathrm{~ms}$ after EECP sessions. This alteration was not found statistically significant with Wilcoxon method, P value: 0.292 , Figure 1.

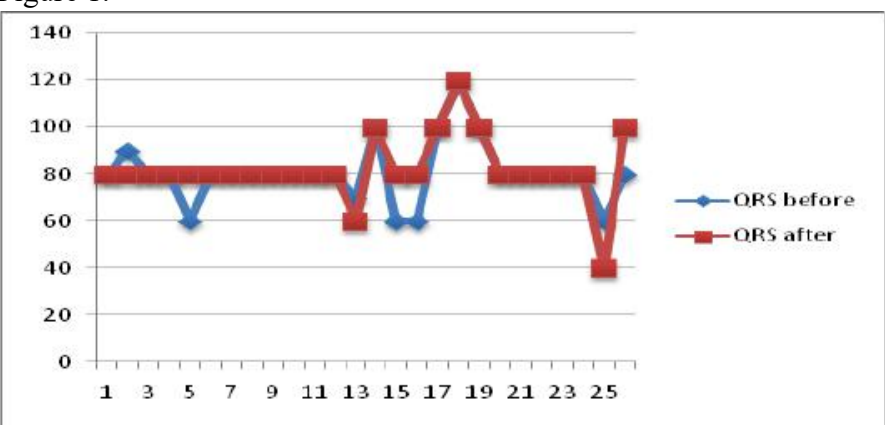

Figure 1: QRS duration (milliseconds) before and after EECP.

\section{Participant Selection}

All participants referred from Tehran Heart Center with functional class II or III in NYHA classification and left ventricular Ejection Fraction $(\mathrm{EF}) \leq 35 \%$ were included in this study. Exclusion criteria were as follows; severe impairment of cardiac valves(moderate to severe stenosis/regurgitation), ventricular arrhythmias, drug resistant Hypertension (HTN), Peripheral arterial disease, pregnancy, NYHA class IV, bleeding or using heparin and warfarin, and patients with Internal Cardioverter Defibrillators (ICDs), cardiac resynchronization therapy with a defibrillator (CRT-d) devices, or pacemaker. The study population included 26 patients that had indication for EECP. Among admitted patients at the EECP center $(n=96), 26$ subjects fulfilled the entrance criteria and were included. All patients had at least 4 ECGs (weekly) and a4 week EECP schedule.

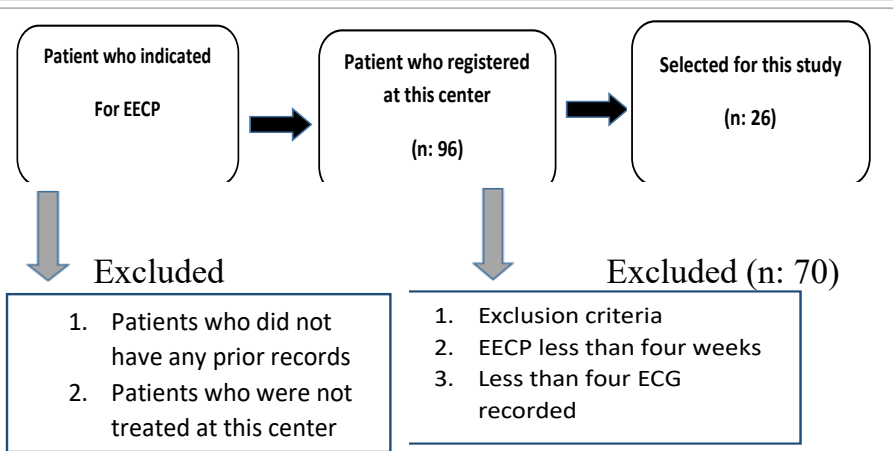

Patient's information including age, gender, weight, past history of diabetes mellitus, hypertension, dyslipidemia and cigarette smoking was gathered before treatment. All patients were assessed by a cardiologist prior to EECP. An echocardiography was performed in order to assess Left Ventricular (LV) function and to exclude significant valvular disease. In each session 12 lead ECG was done and electrocardiographic parameters such as: QT interval (the duration between the initiation of the $\mathrm{Q}$ wave and end of the T wave), QT dispersion (maximum QT interval minus minimum QT interval), QRS duration (A combination of the $\mathrm{Q}$ wave, $\mathrm{R}$ wave and $\mathrm{S}$ wave with normal duration of 0.08 to 0.10 seconds) and PR (measured from the beginning of the $\mathrm{P}$ wave to the beginning of the QRS wave) were evaluated before and after EECP.

QT dispersion (QTd) and corrected QT dispersion (QTcd) were measured by two expert cardiologists. Corrected QT dispersion was calculated by Bazett formula. In case of disagreement on measured QTd, a third cardiologist was involved.

\section{EECP technique}

EECP is done by a technician under the supervision of a cardiologist for duration of one-hour per day in a total of 35 sessions over 7 weeks. The EECP device is consist of three pneumatic cuffs applied to the calves, lower thighs, and upper thighs. A computer-controlled pneumatic system inflates the cuffs in sequence at initiation of diastole, generating approximately 300 $\mathrm{mm} \mathrm{Hg}$ of external pressure. At the onset of systole, the external pressure is released and causes a decline in systolic pressure. Inflation process starts by the electrocardiographic R-wave and continues until the induced retrograde pulse wave improves the cardiac output. For monitoring the pulse pressure wave form, a finger plethysmogram is involved.

\section{Statistical Analysis}

All data were analyzed with SPSS software version 23.0 for Windows (SPSS, Chicago, IL, USA). Evaluation of the normality was done with Kolmogorov-Smirnov test. Normal Variables were analyzed with paired T-test and in case of significant difference from normal distribution, Wilcoxon method was used. $\mathrm{P}$ values $<0.05$ were considered significant.

\section{Results}

Demographic characteristics of 26 patients who were participated in current study are shown in Table 1.

Mean age of subjects was $56 \pm 2.2$ years. Male dominancy was evident ( 21 men vs. 5 women). Changes of certain ECG parameters including PR interval, QRS duration, and QT interval were assessed during 4 weeks of using EECP technique 
for each individual. Baseline ECG was compared with $4^{\text {th }}$ week ECG.

Table 1: Patient Demographics and Baseline Characteristics $(n=26)$.

\begin{tabular}{|l|l|}
\hline Patients Characteristics & Percentage or Mean \\
\hline Age(years) & $56 \pm 2.2$ \\
\hline Gender & $22(84 \%)$ Male \\
& $4(16 \%)$ Female \\
\hline Hypertension & $25(96 \%)$ \\
\hline Diabetes Mellitus & $14(54 \%)$ \\
\hline Hypercholesterolemia & $24(92 \%)$ \\
\hline Current Smoking & $6(23 \%)$ \\
\hline Prior smoking & $23(88 \%)$ \\
\hline Left Ventricular Ejection Fraction & $30 \%$ \\
\hline
\end{tabular}

Mean PR interval was measured $189.6 \mathrm{~ms}$ before EECP and $190.7 \mathrm{~ms}$ after EECP sessions. This alteration was also not found statistically significant, analyzed with paired T test, P value: 0.82 , Figure 2.

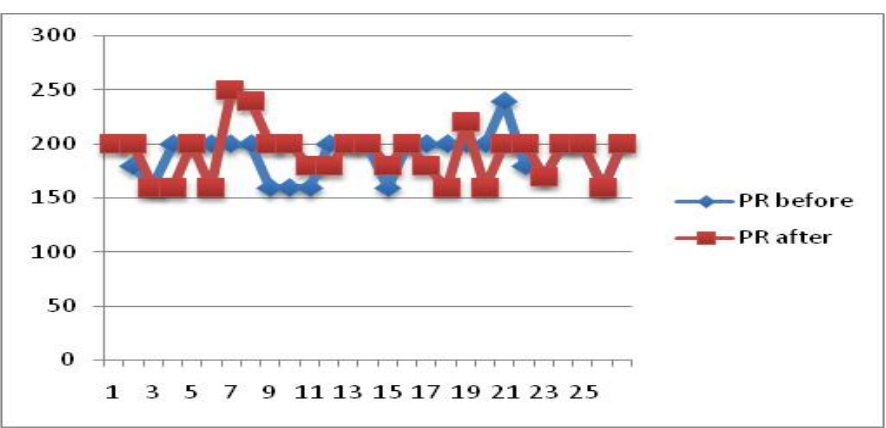

Figure 2: PR interval (milliseconds) before and after EECP.

Most importantly, the differences between mean QT dispersion before and after EECP were measured $65.7 \mathrm{~ms}$ and $64.4 \mathrm{~ms}$ respectively and were compared to each other with $\mathrm{T}$ test. The difference was not statistically significant. (P value: 0.89 ) Figure 3

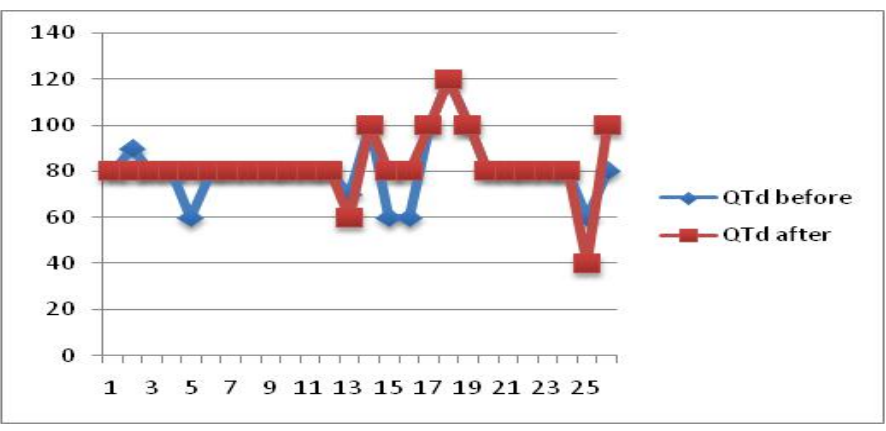

Figure 3: QT dispersion (milliseconds) before and after EECP.

\section{Discussion}

According to statistics, 550,000 new cases of HF are reported annually in U.S $S^{[13]}$. Total financial expenses related to HF was estimated around $\$ 27.9$ billion plus another $\$ 2.9$ billion annually for its treatments ${ }^{[13]}$. Based on previous researches, EECP technique accounts to be an effective rehabilitation method for patients suffering from $\mathrm{HF}$ and poor functional class. Soran $\mathrm{O}$ et al have reported EECP technique to be safe and without any harmful effects during treatment ${ }^{[14]}$. Prospective cohort studies in 2012 on 68 patients have shown that EECP rehabilitation causes significant enhancements in functional class $(p<0.001)$ and LVEF $(\mathrm{p}<0.001)^{[15]}$. In a remarkable study, a large number of patients participated in International EECP Patient Registry were evaluated. Results demonstrate that EECP therapy is a safe and effective treatment for patients with angina or severe LVD who could not undergo CABG or PCI ${ }^{[16]}$. EECP has also positive effects on refractory angina pectoris and quality of life. An interesting review article on this subject was published in 2007 by Manchanda A et al. They investigated the previous articles on effects of using EECP for treatment of angina pectoris ${ }^{[17]}$. One of the major advantages of using EECP is that this technique can enhance the patient's quality of life in various ways which was observed during our study and confirmed by other papers ${ }^{[16]}$. The International Index of Erectile Function (IIEF) scores was compared pre- and post-EECP therapy in a cohort with 120 participants. Results show a significant improvement in erectile function $(p=0.003)^{[18]}$. Campbell et al (2008) have revealed the various effects of EECP technique on brachial blood pressure of patients with normal, low, and high systolic blood pressure. It is declared that EECP technique causes a rise in both systolic and diastolic BP in patients with low BP. On the contrary, it decreases the systolic BP as well as diastolic BP in normal or hypertensive patients ${ }^{[19]}$. Another study by Nichols et al (2006) on patients with refractory angina have shown that EECP treatment results in a major reduction in both peripheral and central systolic pressure along with pulse pressure ${ }^{[20]}$. Different effects of EECP on cardiovascular system are briefed in table 2.

Table 2: EECP's cardiovascular effects.

\begin{tabular}{|l|l|}
\hline Effect & Changes \\
\hline Cardiac after load & Decrease \\
\hline Cardiac Oxygen use & Decrease \\
\hline Coronary flow & Increase \\
\hline Endothelial function & Increase \\
\hline Vasodilatation & Increase \\
\hline Collateral arteries & Increase \\
\hline Arteries perfusion & Increase \\
\hline Ischemia in heart and other organs & Decrease \\
\hline
\end{tabular}

Two different studies on effects of EECP on ECG parameters have shown similar results. Akbarzadeh F. et al (2011) have measured QRS duration, PR interval, QT interval, and QTc interval. Mean QRS duration was declared decreased at the end of study (108 $\pm 18 \mathrm{~ms}$ before and $105 \pm 17 \mathrm{~ms}$ after EECP). QT interval was also reported decreased (from $396 \pm 22 \mathrm{~ms}$ to $391 \pm$ $24 \mathrm{~ms}$ ). QTc interval was changed from $402 \pm 22 \mathrm{~ms}$ to $399 \pm 22$ ms. But PR interval showed a rise. All of these changes were reported statistically insignificant ${ }^{[21]}$. Another work by Henrikson CA et al (2004) was also devoted to measuring the same ECG parameters as mentioned. They have announced that there was no statistically significant changes in assessed markers ${ }^{[22]}$. One of the variables that may have a significant impact on outcome of heart failure patients after different interventions is metabolic syndrome ${ }^{[23,24]}$. In current study, there only available the data was related to blood pressure and diabetes which was not correlated to outcome of patients. However, diabetes may influence the prognosis of heart failure patients with ICDs and CRT-d ${ }^{[25]}$. Comparison between other parameters of metabolic syndrome and patient outcomes or ECG alterations is recommended for future investigations. 
Conclusion: Based on this cross sectional study, EECP did not significantly reduced QT dispersion. However, EECP might improve functional capacity. The effects of EECP on ECG markers have not been yet proven. This may reflect the fact that EECP improves the quality of life in heart failure patients but have no positive influence on prognosis of the disease. We recommend a similar multicenter study with expanded number of participants.

Conflict of Interest: None declared.

Limitations: This is a single center open label non-randomized trial with no control or placebo group study. Drug changes were not monitored during the trial. Most of the participants did not complete 35 sessions. Small sample size is another limitation of current work. We recommend multicenter approach with a larger sample size for future investigations. Not all of the items related to metabolic syndrome, which may affect the outcome of the patients, were included in our collected data. 6 and 12 month follow-up of patients for the number of hospital admissions, functional class changes, and ECG parameters alterations is recommended for future studies.

Acknowledgements: The authors would like to thank Students' Scientific Research Center (SSRC) and Cardiac Primary Prevention Research Center for their support and the Research Development Center of Tehran Heart Center for their technical assistance. This study is supported in all aspects by Tehran University of Medical Sciences (TUMS).

\section{References}

1. Strobeck, J.E. Enhanced external counterpulsation in congestive heart failure: possibly the most potent inodilator to date. (2002) Congest Heart Fail 8(4): 201-203.

Pubmed $\mid$ Crossref $\mid$ Others

2. Stys, T., Lawson, W.E., Hui, J.C., et al. Acute hemodynamic effects and angina improvement with enhanced external counterpulsation. (2001) Angiology 52(10): 653-658.

Pubmed $\mid$ Crossref $\mid$ Others

3. Sharma, U., Ramsey, H.K., Tak, T. The role of enhanced external counter pulsation therapy in clinical practice. (2013) Clin Med Res 11(4): 226-232. Pubmed $\mid$ Crossref $\mid$ Others

4. Bonetti, P.O., Holmes, D.R., Lerman, A., et al. Enhanced external counterpulsation for ischemic heart disease: what's behind the curtain? (2003) J Am Coll Cardiol 41(11): 1918-1925.

Pubmed $\mid$ Crossref $\mid$ Others

5. Lawson, W.E., Kennard, E.D., Holubkov, R., et al. Benefit and safety of enhanced external counterpulsation in treating coronary artery disease patients with a history of congestive heart failure. (2001) Cardiology 96(2): 78-84.

Pubmed $\mid$ Crossref $\mid$ Others

6. Feldman, A.M., Silver, M.A., Francis, G.S., et al. Enhanced external counterpulsation improves exercise tolerance in patients with chronic heart failure. (2006) J Am Coll Cardiol 48(6): 1198-1205.

Pubmed $\mid$ Crossref $\mid$ Others

7. Campbell, R.W.F., Gardiner, P., Amos, P.A., et al. Measurement of the QT interval. (1985) Eur Heart J 6 (Suppl D): 81-83.

Pubmed $\mid$ Crossref $\mid$ Others

8. Day, C.P., McComb, J.M., Campbell, R.W. QT dispersion: an indication of arrhythmia risk in patients with long QT intervals. (1990) Br Heart J 63(6): 342-344.

Pubmed $\mid$ Crossref $\mid$ Others

9. Day, C.P., McComb, J.M. Campbell, R.W. QT dispersion in sinus beats and ventricular extrasystoles in normal hearts. (1992) Br Heart J 67(1): 39-41.

Pubmed $\mid$ Crossref $\mid$ Others
10. Higham, P.D., Furniss, S.S., Campbell, R.W. QT dispersion and components of the QT interval in ischaemia and infarction. (1995) Br Heart J 73(1): 32-36.

Pubmed $\mid$ Crossref $\mid$ Others

11. Nishiyama, Y., Maeda, H., Tanaka, M., et al. Effect of physical training on corrected QT dispersion in patients with nonischemic heart failure. (2004) Circ J 68(10): 946-949.

Pubmed $\mid$ Crossref $\mid$ Others

12. Fujimoto, S.1., Uemura, S., Tomoda, Y., et al. Effects of exercise training on the heart rate variability and QT dispersion of patients with acute myocardial infarction. (1999) Jpn Circ J 63(8): 577-582.

Pubmed | Crossref | Others

13. American Heart Association. Heart Disease and Stroke Statistics. Dallas, TX: American Heart Association. 2005.

Pubmed $\mid$ Crossref $\mid$ Others

14. Soran, O., Fleishman, B., Demarco, T., et al. Enhanced external counterpulsation in patients with heart failure: a multicenter feasibility study. (2002) Congest Heart Fail 8(4): 204-208, 227.

Pubmed $\mid$ Crossref $\mid$ Others

15. Kozdag, G., Ertaş, G., Aygün, F., et al. Clinical effects of enhanced external counterpulsation treatment in patients with ischemic heart failure. (2012) Anadolu Kardiyol Derg 12(3): 214-221.

Pubmed $\mid$ Crossref $\mid$ Others

16. Soran, O., Kennard, E.D., Kfoury, A.G., et al. Two-year clinical outcomes after enhanced external counterpulsation (EECP) therapy in patients with refractory angina pectoris and left ventricular dysfunction (report from The International EECP Patient Registry). (2006) Am J Cardiol 97(1): 17-20.

Pubmed $\mid$ Crossref $\mid$ Others

17. Manchanda, A., Soran, O. Enhanced external counterpulsation and future directions: step beyond medical management for patients with angina and heart failure. (2007) J Am Coll Cardiol 50(16): 1523-1531.

Pubmed | Crossref | Others

18. Lawson, W.E., Hui, J.C., Kennard, E.D., et al. Effect of enhanced external counterpulsation on medically refractory angina patients with erectile dysfunction. (2007) Int J Clin Pract 61(5): 757-762.

Pubmed $\mid$ Crossref $\mid$ Others

19. Campbell, A.R., Satran, D., Zenovich, A.G., et al. Enhanced external counterpulsation improves systolic blood pressure in patients with refractory angina. (2008) Am Heart J 156(6): 1217-1222.

Pubmed $\mid$ Crossref $\mid$ Others

20. Nichols, W.W., Estrada, J.C., Braith, R.W., et al. Enhanced external counterpulsation treatment improves arterial wall properties and wave reflection characteristics in patients with refractory angina. (2006) J Am Coll Cardiol 48(6): 1208-1214.

Pubmed | Crossref $\mid$ Others

21. Akbarzadeh, F., Salekzamani, Y., Beigzdeh, A.S. The Effects of Enhanced External Counterpulsation on Cardiac Electrophysiologic Properties of Patients with Ischemic Heart Disease and Refractory Angina at Function Class II-III. (2011) J Cardiovasc Thorac Res 3(2): 49-51.

Pubmed $\mid$ Crossref $\mid$ Others

22. Henrikson, C.A., Chandra-Strobos, N. Enhanced external counterpulsation therapy: significant clinical improvement without electrophysiologic remodeling. (2004) Ann Noninvasive Electrocardiol 9(3): 265-269.

Pubmed $\mid$ Crossref $\mid$ Others

23. Sardu, C., Carreras, G., Katsanos, S., et al. Metabolic syndrome is associated with a poor outcome in patients affected by outflow tract premature ventricular contractions treated by catheter ablation. (2014) BMC Cardiovasc Disord 14: 176.

Pubmed | Crossref | Others

24. Sardu, C., Santamaria, M., Funaro, S., et al. Cardiac electrophysiological alterations and clinical response in cardiac resynchronization therapy with a defibrillator treated patients affected by metabolic syndrome. (2017) Medicine 96(14): e6558.

Pubmed $\mid$ Crossref $\mid$ Others

25. Sardu, C., Barbieri, M., Santamaria, M., et al. Multipolar pacing by cardiac resynchronization therapy with a defibrillators treatment in type 2 diabetes mellitus failing heart patients: impact on responders rate, and clinical outcomes. (2017) Cardiovascular Diabetology 16(1): 75.

Pubmed | Crossref | Others 